\section{P08.21 SEX AND PELVIC INFLAMMATORY DISEASE: WHAT'S THE RELATIONSHIP? CASE-CONTROL STUDY}

${ }^{1} \mathrm{~N}$ Low ${ }^{*},{ }^{2} \mathrm{~A}$ Hegazi, ${ }^{2} \mathrm{~S}-\mathrm{Y}$ Chan, ${ }^{2} \mathrm{P}$ Hay, ${ }^{1,3} \mathrm{SA}$ Herzog. ${ }^{1}$ Institute of Social and Preventive Medicine, University of Bern, Bern, Switzerland; ' ${ }^{2}$ Courtyard Clinic, St. George's NHS Foundation Trust, London, UK; ${ }^{3}$ Institute for Medical Informatics, Statistics and Documentation, Medical University of Graz, Austria

\subsection{6/sextrans-2015-052270.367}

Background Pelvic inflammatory disease (PID) results from infection ascending to the upper female genital tract. The timing of progression of untreated infections is poorly understood and difficult to study prospectively. We investigated temporal relationships between recent sexual partnerships and PID caused by the sexually transmitted infections (STI) Chlamydia trachomatis or Neisseria gonorrhoeae.

Methods We did a case-control study, using case records. Cases were women with clinically diagnosed PID and a positive C. trachomatis or N. gonorrhoeae test result. Control groups were women who presented on the same day with: control1) clinical PID and negative test results; control $^{2}$ ) no clinical PID and negative test results; control3) uncomplicated C. trachomatis or control4) uncomplicated N. gonorrhoeae infection. We used survival methods for statistical analysis.

Results We analysed data from 356 women: 72 cases, 83 control1, 75 control $^{2}, 68$ control3 and 58 control4. Cases and women in control3 and control4 were younger than women in control1 or control $^{2}(p<0.001)$, intrauterine device use and dates of last menstruation before attendance were similar in all groups. Women with chlamydial or gonococcal PID (cases) had more recently changed sexual partners (median 154 days, IQR 61-736) than those with clinical PID but no infection (control1, median 367 days, IQR 94-1419, $\mathrm{p}=0.082$ ). The time from the start of the most recent sexual partnership to symptom onset was shorter in cases (median 121 days, IQR 47-695) than control1 (median 366 days, IQR 104-1552, crude hazard ratio, HR $0.65,95 \%$ CI $0.46-0.92)$. After adjusting for age, this association was weakened (adjusted HR 0.81, 95\% CI 0.56-1.17).

Conclusion Differences in the course of STI- and non-STI associated PID were mainly due to age. Further studies to elucidate the course of acute STI and ascending infection will help to understand the impact of screening and treatment interventions on PID prevention.

Disclosure of interest No funding was received for this study.

\section{P08.22 HOW HIGH IS HIGH RISK? SEXUAL BEHAVIOUR AND CHLAMYDIA INFECTIONS IN WOMEN ATTENDING GENITOURINARY MEDICINE CLINICS}

${ }^{1} \mathrm{~F}$ Krauer${ }^{*},{ }^{2} \mathrm{~S}$ Oakley-Mudge, ${ }^{3} \mathrm{MG}$ Brook, ${ }^{4} \mathrm{JA}$ White, ${ }^{1} \mathrm{~N}$ Low, ${ }^{2} \mathrm{HH}$ Lee. ${ }^{1}$ Institute of Social and Preventive Medicine, University of Bern, Bern, Switzerland; ${ }^{2}$ Diagnostics for the Real World, Cambridge, UK; ${ }^{3}$ Patrick Clements Clinic, Central Middlesex Hospital, London, UK; ${ }^{4}$ Department of Genitourinary Medicine, Guy's and St Thomas' NHS Foundation Trust, London, UK

\subsection{6/sextrans-2015-052270.368}

Introduction Population based surveys provide valuable data for the general population but most testing for sexually transmitted infections (STI) is done in health care settings. The objective of this cross-sectional study was to describe sexual behaviour characteristics of women attending genitourinary medicine (GUM) clinics and compare them with nationally representative data.
Methods This study was conducted from January 2014 to March 2015 in two London clinics. Women were eligible if they were aged 16-29 years and able to provide informed consent. Participants completed a web-based questionnaire that included several questions from the third British National Survey of Sexual Attitudes and Lifestyles (Natsal-3). We compared clinic attenders with women aged 16-24 years in the general population, surveyed in Natsal-3 in 2010-2011.

Results We enrolled 1808 women, of whom 1806 provided clinical data and 1792 completed the questionnaire. Most (61.9\%) were 16-24 years old and from white ethnic groups (48.8\%). Four fifths reported having ever been tested for chlamydia with an average of 3.3 (SD 1.8) tests per participant. One third had ever been diagnosed with chlamydia. Compared with women age 16-24 years in Natsal-3, clinic attenders were more likely to report $\geq 5$ lifetime partners $(47.0 \%$ vs. $37.3 \%, \mathrm{p}<0.001)$ and $\geq 2$ partners in the past year $(51.8 \%$ vs. $26.7 \%$, p $<0.001)$, but fewer sex acts in the past four weeks $(4.1$, SD 4.7 vs. 5.8, SD $6.6, \mathrm{p}<0.001) .43 \%$ of clinic attenders had had at least one concurrent relationship.

Conclusion This study provides detailed data about differences in sexual activity and practices between women attending clinics and those in the general population. These results can be used in studies to understand the impact of interventions such as pointof-care testing to improve chlamydia screening outcomes.

Disclosure of interest statement This study was funded by the UK Technology Strategy Board; HHL is an equity holder of the company Diagnostics for the Real World.

\section{P08.23 SERO-EPIDEMIOLOGICAL ASSESSMENT INDICATES HIGH PREVALENCE OF C. TRACHOMATIS IN SAMOAN WOMEN WITH INFERTILITY}

${ }^{1} \mathrm{~S}$ Menon*, ${ }^{1} \mathrm{~S}$ Stansfield, ${ }^{2} \mathrm{M}$ Walsh, ${ }^{3} \mathrm{E}$ Hope, ${ }^{4} \mathrm{~L}$ Isaia, ${ }^{2} \mathrm{~A}$ Righarts, ${ }^{5} \mathrm{~T}$ Niupulusu, ${ }^{6}$ SVA Temese, ${ }^{7} \mathrm{~L}$ losefa, ${ }^{3} \mathrm{~L}$ Auvaa, S Tapelu, MF Motu, ${ }^{8} \mathrm{~T}$ Suaalii-Sauni, ${ }^{9} \mathrm{P}$ Timms, ${ }^{2} \mathrm{P}$ Hill, ${ }^{1}$ WM Huston. ${ }^{1}$ Institute of Health and Biomedical Innovation, Queensland University of Technology, Brisbane, Australia; ${ }^{2}$ Centre for International Health, University of Otago, Dunedin, New Zealand; ${ }^{3}$ National University of Samoa, Samoa; ${ }^{4}$ National Health Service Laboratory Division, Samoa; ${ }^{5}$ Samoa Family Health Association, Samoa; ${ }^{6}$ Samoa AIDS Foundation, Samoa; ${ }^{7}$ Samoa National Council of Churches, Samoa; ${ }^{8}$ Victoria University of Wellington, New Zealand; ${ }^{9}$ Faculty of Science, Health, Education and Engineering, University of the Sunshine Coast, Australia; ${ }^{8}$ Queensland University of Technology, Australia

\subsection{6/sextrans-2015-052270.369}

Introduction Chlamydia trachomatis (CT) is one of the most common bacterial sexually transmitted infections in the world. Due to the asymptomatic nature of the disease, the infection is frequently undiagnosed resulting in the development of serious sequelae such as pelvic inflammatory disease, ectopic pregnancy and tubal infertility in women. The prevalence of CT infection in Samoa was previously estimated to be $30.9 \%$, based on pregnant women attending antenatal clinics. The high prevalence of CT infection may imply a high probability of increased risk of sequelae such as infertility in Samoan women.

Methods Serological prediction of CT infertility as indicated by titers of serum antibodies to CT in infertile women was conducted using a series of commercial tests such as MEDAC and ANilabsystems serology kits. The correlation between selfreported infertility and epidemiological factors to serologically predicted CT infertility was determined. Self reported infertility in women was defined based on their patient history as the inability to get pregnant after trying for more than 1 year. 